\title{
Artificial Neural Networks Application in Modal Analysis of Tires
}

\author{
P. Koštial ${ }^{1}$, Z. Jančíková ${ }^{2}$, D. Bakošová ${ }^{3}$, J.Valíček $^{4,5}$, M. Harničárová ${ }^{4,6}$, I. Špička ${ }^{2}$ \\ ${ }^{1}$ Department of Material Engineering, Faculty of Metallurgy and Materials Engineering, VŠB-Technical University of \\ Ostrava, 17. listopadu 15/2172, 70833 Ostrava-Poruba, Czech Republic, pavel.kostial@vsb.cz \\ ${ }^{2}$ Department of Automation and Computer Science in Metallurgy, Faculty of Metallurgy and Materials Engineering, \\ VŠB-Technical University of Ostrava, 17. listopadu 15/2172, 70833 Ostrava-Poruba, Czech Republic, \\ zora.jancikova@vsb.cz, ivo.spicka@vsb.cz \\ ${ }^{3}$ Department of Physical Engineering of Materials, Faculty of Industrial Technologies, University of Alexander Dubček in \\ Trenčín, I. Krasku 491/30, 02001 Púchov, Slovak Republic \\ ${ }^{4}$ Institute of Physics, Faculty of Mining and Geology, VŠB-Technical University of Ostrava, 17. listopadu 15/2172, \\ 70833 Ostrava-Poruba, Czech Republic, jan.valicek@vsb.cz \\ ${ }^{5}$ RMTVC, Faculty of Metallurgy and Materials Engineering,VŠB-Technical University of Ostrava, 17. listopadu 15/2172, \\ 70833 Ostrava-Poruba, Czech Republic, jan.valicek@vsb.cz \\ ${ }^{6}$ Nanotechnology Centre, VŠB-Technical University of Ostrava, 17. listopadu 15/2172, \\ 70833 Ostrava-Poruba, Czech Republic, marta.harnicarova@vsb.cz
}

\begin{abstract}
The paper deals with the application of artificial neural networks (ANN) to tires' own frequency (OF) prediction depending on a tire construction. Experimental data of OF were obtained by electronic speckle pattern interferometry (ESPI). A very good conformity of both experimental and predicted data sets is presented here. The presented ANN method applied to ESPI experimental data can effectively help designers to optimize dimensions of tires from the point of view of their noise.
\end{abstract}

Keywords: ESPI, modal analysis, neural networks, tires.

\section{INTRODUCTION}

$\mathrm{A}$ TIRE IS DESIGNED to satisfy a number of functional requirements. The rubber structure must work reliably in a large spectrum of dynamic conditions - from static loadings to high frequency exciting of mechanical vibrations. From the viewpoint of mechanics there is a complicated dynamic system, where rubber material nonlinearities, as well as composite anisotropic structure, have to be taken into account. The performance requirements of the tire system cannot be adequately designed without knowledge of its real dynamic characteristics, where the experimental measurements are necessary. This holds also for the measurement of natural frequency modes/shapes and damping properties as well.

From the practical point of view the knowledge of natural tire frequencies gives the designers a chance to change the tire construction in such a way so that the driving comfort connected with tire noise is optimized [1].

The non-contact and full-field measurement allows the calculation of the three-dimensional distribution of a displacement of the object under test as a response to a mechanical or thermal loading [2]. Electronic speckle pattern interferometry can serve as a tool of an easy determination of Poisson's ratio v, Young's modulus E and shear modulus $\mathrm{G}$ from the measured resonant frequencies.

Subsequently, it is, for example, possible to make various computed models on the basis of the values determined by ESPI [2], [3]. ESPI is an optical technique which enables interferometric measurements of surface displacements on almost any surface and material.
Work [4] presents the ESPI application for non-destructive measurement in situ displacement fields in micro-systems. A thermal vacuum chamber was designed to induce thermal treatments, including annealing. From the identification of the residual-stress-free state, they quantitatively modelled thermal strains/stress fields, relaxation stresses during annealing, and residual stress fields. Both electronic shearography and ESPI techniques are capable of detecting discontinuities, however, little or no attention has been paid to the determination of the severity or effect of such a discontinuity that might be acceptable. The same discontinuity would become a defect only if its size, position direction, and stress environment indicate a performance risk [5].

Neural networks use a distributed parallel processing of the information during practicing of calculations, it means that information recording, processing and transferring are carried out by means of the whole neural network rather then by means of particular memory places. Learning is a basic and essential feature of neural networks. Knowledge is recorded especially through strength of linkages between particular neurons. Linkages between neurons leading to "correct answer" are strengthened and linkages leading to "wrong answer" are weakened by means of repeated exposure of examples describing the problem area. A complex of submitted examples creates a so called training set [6].

A capability to learn from examples and ability to describe well also non-linear dependences is a big advantage of neural networks. A disadvantage is the fact, that the size of error, which is strongly dependent on network parameters 
and on a training set data quality, cannot be generally estimated in advance. The design of a structure and parameters of the neural network are always connected with some experiences. The experience, intuition and experiments are also important for the optimization of the neural network. Neural networks, which are universal function approximators, especially Backpropagation type networks utilizing their learning (adaptation) Backpropagation algorithm, are suitable essentially for all types of predictions. This algorithm is suitable for multilayer feedforward network learning, which is created minimally by three layers of neurons: input, output and at least one inner (hidden) layer (Fig.1.).

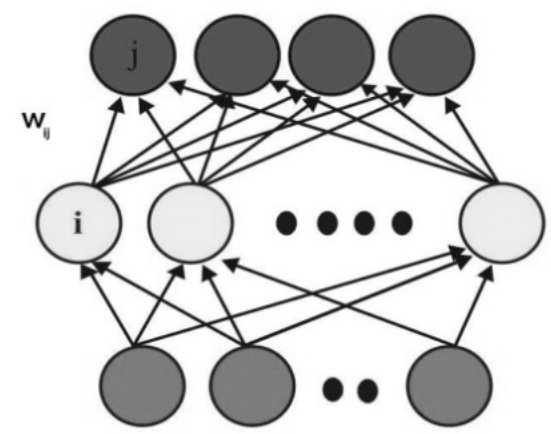

Fig.1. Topology of multilayer feedforward neural network.

Between two adjoining layers there is always the so called total connection of neurons, thus each neuron of the lower layer is connected to all neurons of the higher layer. Learning in the neural network is realized by setting the values of synaptic weights between neurons, biases or inclines of activation functions of neurons. The adaptation at networks with Backpropagation algorithm is called „supervised learning“, when the neural network learns by a comparison of the actual and the required output. The algorithm tries to accomplish a minimal difference between required value and value on the network output by a gradual setting of synaptic weights [7].

The rate of inaccuracy between predicted value of neural network output and actual value of object output represents a prediction error. In technical applications the error is mainly represented by the following parameters with obvious labelling: SSE, R-square, Adjusted R-Square, Root Mean Squared Error RMSE [8].

In practice, the problems solved by neural networks are most often realized by means of a computer program. Each program unit dealing with the functioning of a neural network can be divided into several concurring parts. These parts correspond to the procedures that are used when working with neural networks. It is a synthesis of the neural network itself, the neural network determination, followed by the actual state when an algorithm solving the problem is designed thanks to the outcomes of the previous operations.

For a draft of measures for an improvement of the steelwork production quality neural networks can be successfully applied in such case, because they are especially suitable for an approximation of relations between various sensor-based data, particularly between unstructured data with a high degree of nonlinearity and a big scale of uncertainty [9], [10].

In this paper we present results of ESPI measurements of tires' $O F$ depending on tire construction parameters as well as $A N N$ application of ESPI data for $O F$ and $O A$ prediction depending on chosen tire construction parameters.

\section{EXPERIMENTAL PROCEDURE}

The experimental procedure of the ESPI technique is performed as follows. Tires are excited by a loudspeaker in radial and axial directions. First, a reference image is taken, after the specimen vibrates, then the second image is taken, and the reference image is subtracted by the image processing system. If the vibrating frequency is not the resonant frequency, only randomly distributed speckles are displayed and no fringe patterns will be shown. However, if the vibrating frequency is within the scope of the resonant frequency, stationary distinct fringe patterns will be observed. Then the function generator is carefully and slowly turned, the number of fringes will increase and the fringe pattern will become clearer, as the resonant frequency is being approached. The resonant frequencies and corresponding mode shapes can be determined at the same time, using the ESPI optical system presented in Fig.2.
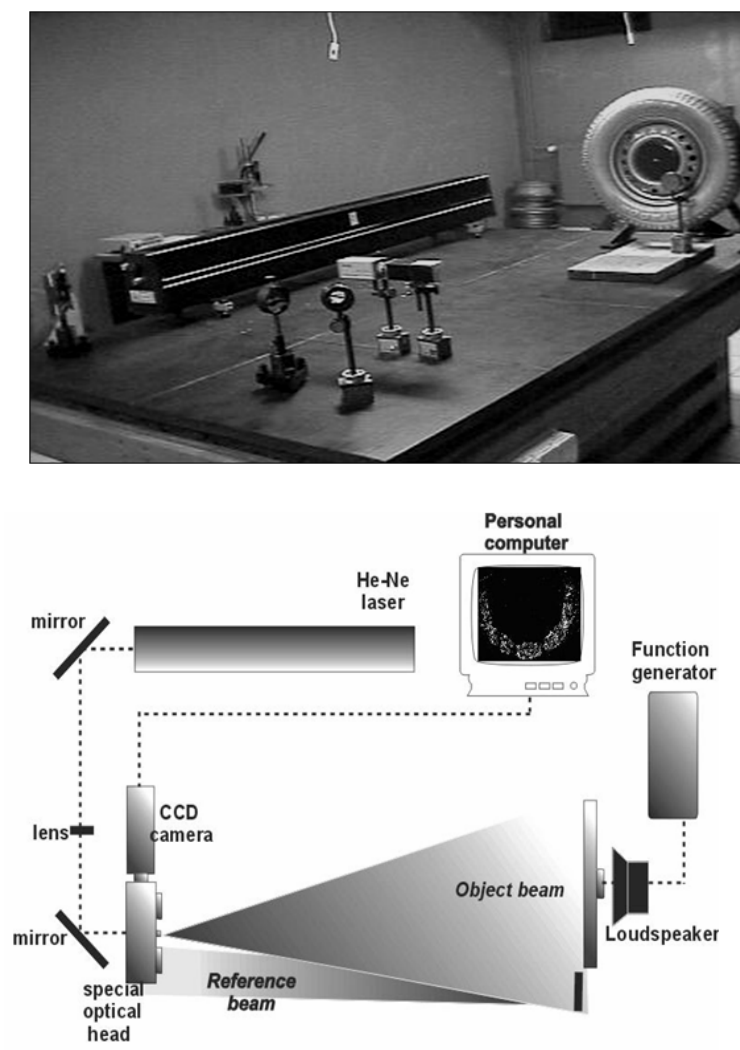

Fig.2. Real experimental view on ESPI apparatus (above) and its schema (down).

\section{ANN AND ESPI DATA ANALYSIS}

In the first step, we tested the whole accessible spectrum of vibration modes, their frequencies in both radial (label R) and axial direction (label A). The results were obtained for the tire Matador MP 42 195/70 R14 91H (Table 1.). 
Table.1. ESPI tire modal analyse data set for the tire MP 42 195/70 R14 91H.

\begin{tabular}{|c|c|}
\hline Mode & Frequency [Hz] \\
\hline Axial $1^{\text {st }}$ mode & 41 \\
\hline Axial $2^{\text {nd }}$ mode & 90 \\
\hline Axial $3^{\text {rd }}$ mode & 150 \\
\hline Radial $1^{\text {st }}$ mode & 90 \\
\hline Radial $2^{\text {nd }}$ mode & 113 \\
\hline Radial $3^{\text {rd }}$ mode & 133 \\
\hline Radial $4^{\text {th }}$ mode & 157 \\
\hline Radial $5^{\text {th }}$ mode & 183 \\
\hline Radial $6^{\text {th }}$ mode & 202 \\
\hline
\end{tabular}

Table 2. presents results of OF (axial modes A1, A2, A3, radial modes $\mathrm{R} 1, \mathrm{R} 2, \mathrm{R} 3$ ) of three different producers for tires of the same dimensions. Differences in technological realization of tire cavities (reflected by different OF) which have the same volume in all cases under investigation are clearly seen.

Table. 2. Frequency versus chosen modes of three different tire producers labelled as $\mathrm{C}, \mathrm{D}, \mathrm{M}$ of the same tire parameters 205/55 R16 91W.

\begin{tabular}{|c|c|c|c|}
\hline Mode & C [freq. Hz] & D [freq. Hz] & M [freq. Hz] \\
\hline A1 & 64 & 65 & 81 \\
\hline A2 & 101 & 100 & 96 \\
\hline A3 & 169 & 180 & 175 \\
\hline R1 & 90 & 90 & 66 \\
\hline R2 & 120 & 125 & 122 \\
\hline R3 & 148 & 131 & 140 \\
\hline
\end{tabular}

To study the dynamic changes of the $O F$ mode spectrum on the same tire we have changed the tire inflation and investigated the changes registered in $O F$. The results for the first four modes are presented in Fig.3.

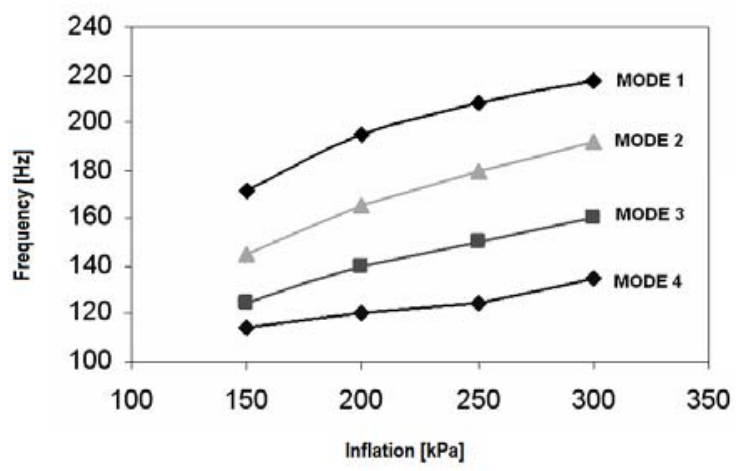

Fig.3. The OF changes versus inflation.

As expected, the mode frequency decreases with decreasing inflation for a tire excitation in radial direction. Qualitatively, we have obtained very close results at the axial excitation of the tire. Fig.4. shows a real view on ESPI mode shape of the sixth mode generated at different inflations.
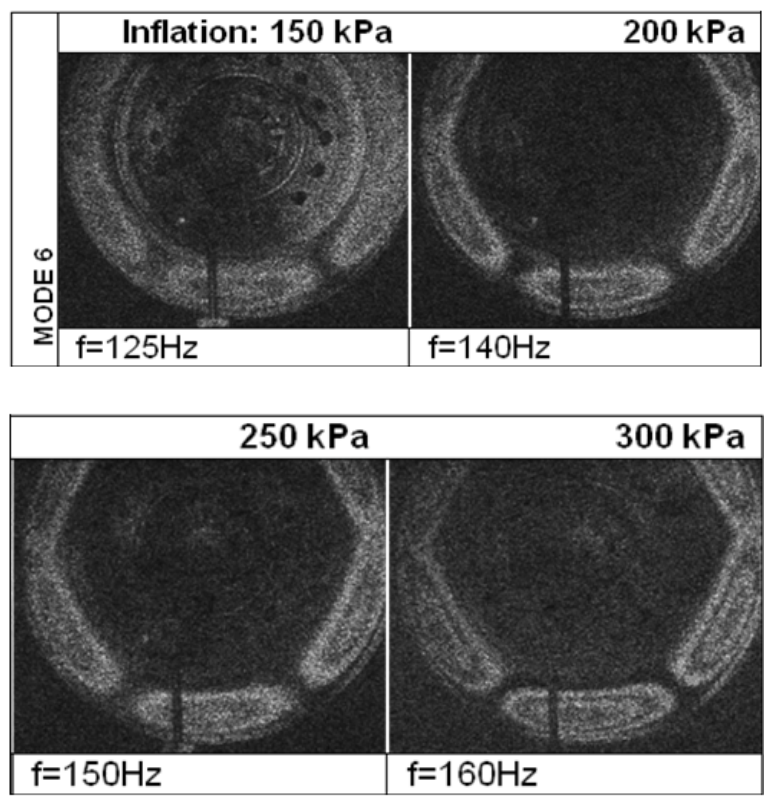

Fig.4. Experimental ESPI results for mode 6 at different inflations.

To obtain the qualitative evaluation of the mode structure we use FEM to model tire vibrations in MARC software environment. The Mooney constants were used as material parameters and rubber plasticity model for big deformation was applied. The simulation was realized at following assumptions:

- Material properties are defined by Mooney constants.

- We considered the simulated tire without internal reinforcement (cords).

- The Rubber Plasticity Procedure with big deformations was used.

- For the calculation, we have used Marc Designer software.

Both experimental and simulation results are in very good qualitative harmony. FEM simulation was realized on a simple model of a tire without a rim, breakers and other parts of a real tire. Nevertheless, for quantitative match of the experiment and simulation results, it is necessary to know the details of the tire material composition and construction, which is very difficult to obtain because producers usually do not disclose this information. The shape of exited and simulated modes is presented in Fig.5.

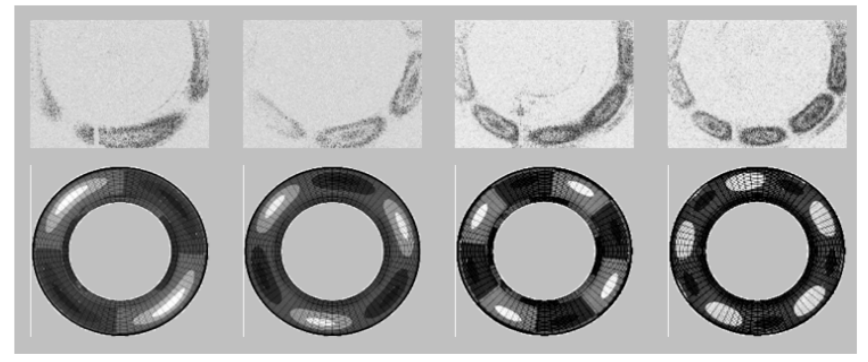

Fig.5. Measured by ESPI (upper part) and simulated by FEM (lower part) vibration modes. 
After these experiments we tried to find experimental conditions for the appearance of OF pattern obtained by ESPI at real tire rotation on a testing machine. Fig.6. shows the standing wave packets (right) obtained by the Aramis system (measured changes of a displacement) at the velocity of $210 \mathrm{~km} / \mathrm{h}$. The details of this test are visible in the bottom part of Fig. 6 .
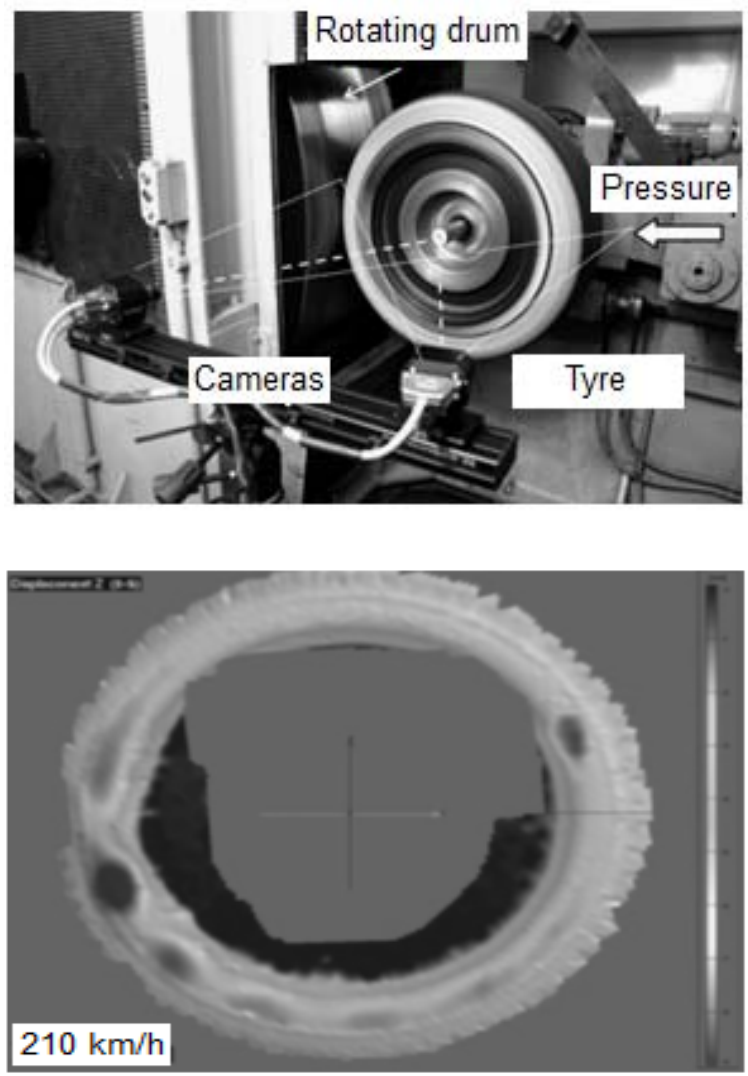

Fig.6. Measured by ESPI (upper part) and simulated by FEM (lower part) vibration modes.

In the next part of the contribution we present comparison of measured and predicted own frequencies of tires. An application that expects the tire parameters to serve as the input values was created as a sample of implementation. The parameters are the nominal width, profile number, rim diameter, load index and speed index. These parameters are used as input to the algorithm. The output is a predicted value of radial frequency and amplitude.

The acquired database for prediction of noise contained 83 cases. Due to the absence of values in case of some variables, the database had been modified and included only 69 cases. These facts were subsequently adjusted to a form suitable for the application of neural network. The whole database was divided into the data to be used for the network learning (training and validation set) and the data to be later used to check the prediction accuracy, i.e. the generalization ability of the neural network (test set). Artificial neural networks were subsequently designed and trained on the basis of the adjusted data. The best prediction results were achieved by a three-layer perceptron neural network of 5-11-2 topology (5 input neurons, 11 in the hidden layer and 2 outputs). Results are presented in graphic form in Fig.7. [11].

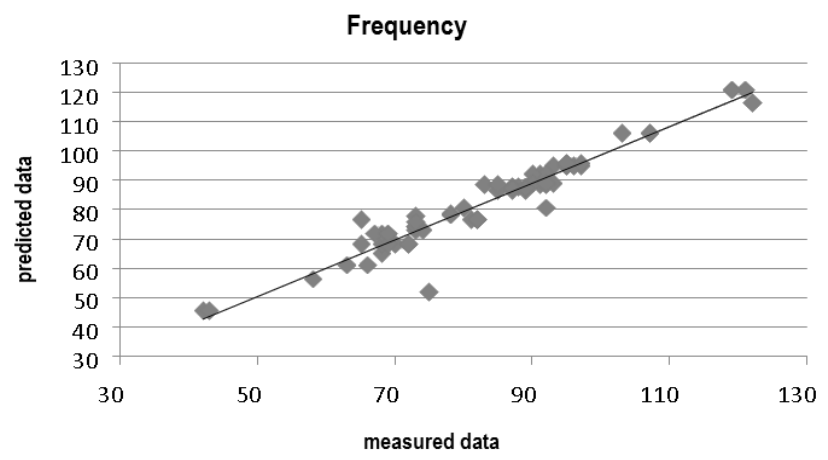

Fig.7. Comparison of measured and predicted frequency data obtained by ESPI and $A N N$.

To test the reliability of chosen network we have evaluated next 120 tyres and we obtained also the same network configuration with statistical data $S S E=526.127, R M S$ $=1218, R^{2}=0.985$. For a third group containing 98 tires we obtain values $S S E=723.221, R M S=962, R^{2}=0.988$ at the same network configuration. These results clearly show reliability of chosen network.

Further input parameters of neural network were enlarged. Radial static stiffness, circumferential static stiffness, lateral static stiffness, static torsional stiffness have been added as an input data.

In Fig. 8 dependences of radial frequency on stiffnesses defined above are presented. The results of this analysis show independency of the radial frequency on the static torsional stiffness (Fig. 8d). In other presented cases the assumed dependence is decreasing.

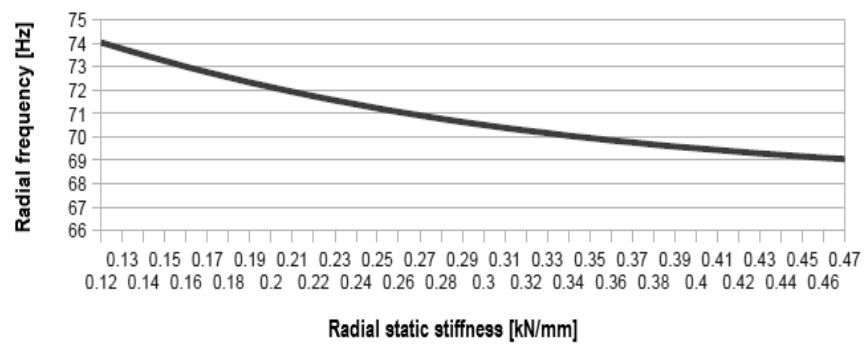

Fig.8a. Dependence of the radial frequency on the radial stiffness.

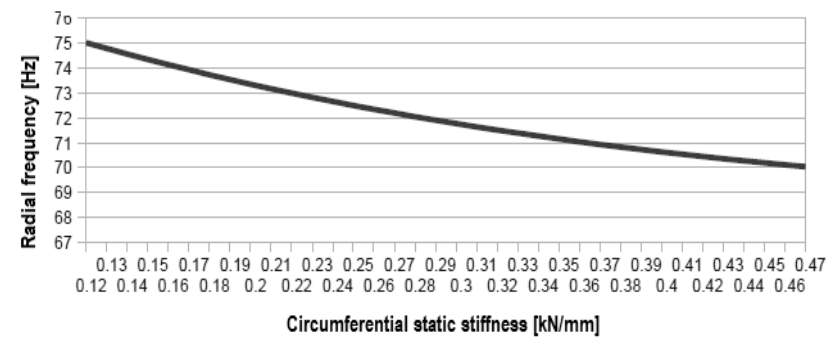

Fig.8b. Dependence of the radial frequency on circumferential static stiffness. 


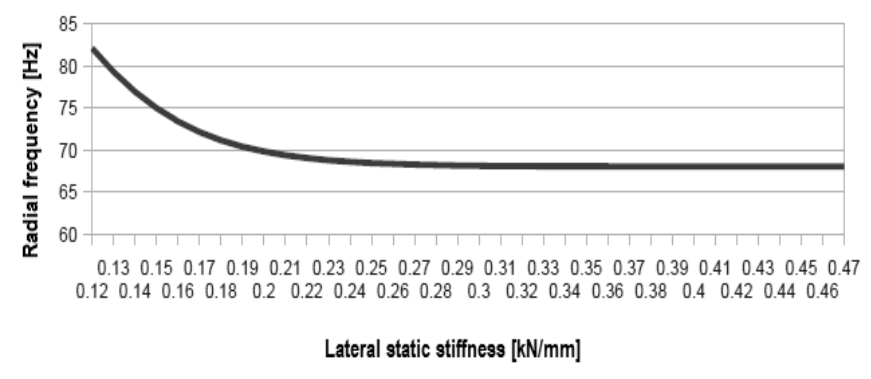

Fig.8c. Dependence of the radial frequency on lateral static stiffness.

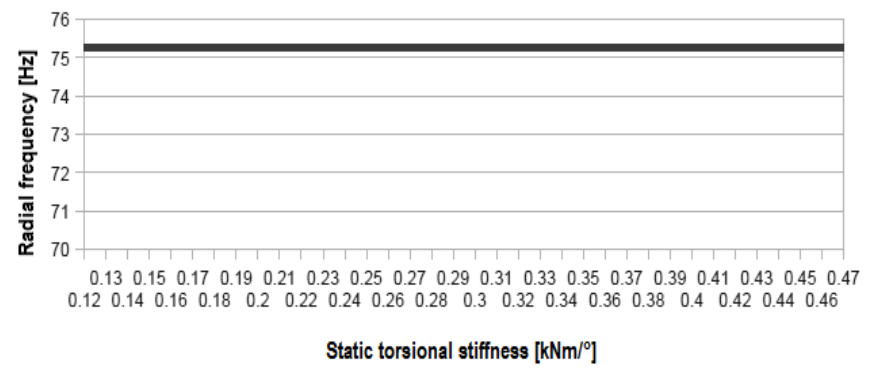

Fig.8d. Dependency of the radial frequency on static torsional stiffness.

In Fig.9. are dependences of radial amplitude versus the above mentioned stiffnesses. The results of this analysis show independency of the radial amplitude on the static torsional stiffness. These results were obtained for a tire with the width of $155 \mathrm{~mm}$, profile number 55, rim diameter 14 inches, load index 80, velocity index $H$.

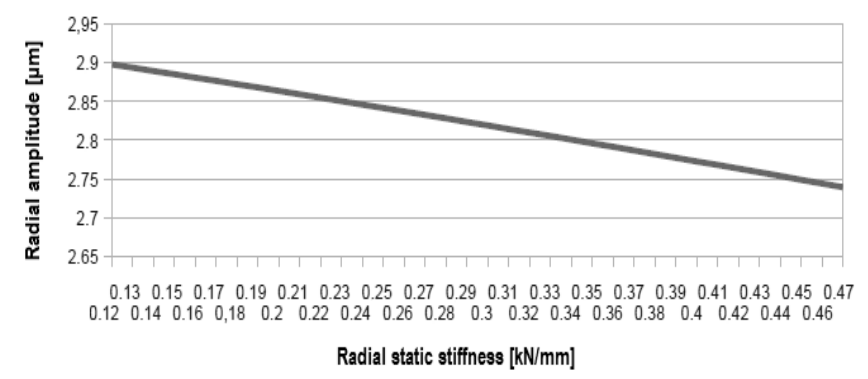

Fig.9a. Dependence of the radial amplitude on the radial stiffness.

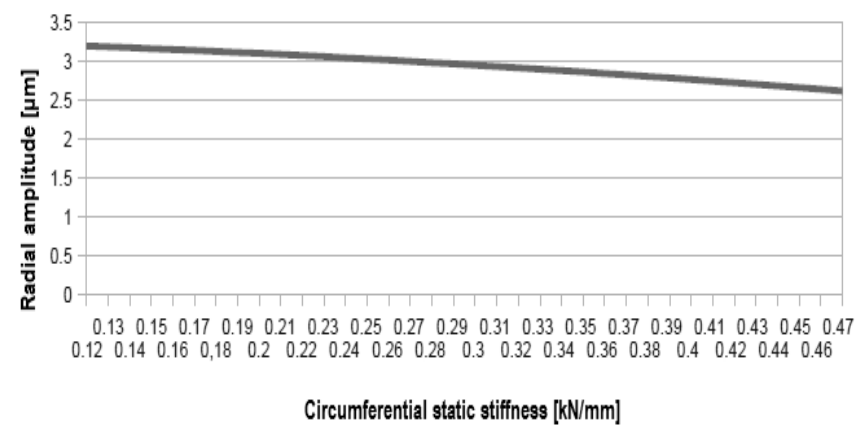

Fig.9b.Dependence of the radial amplitude on circumferential static stiffness.

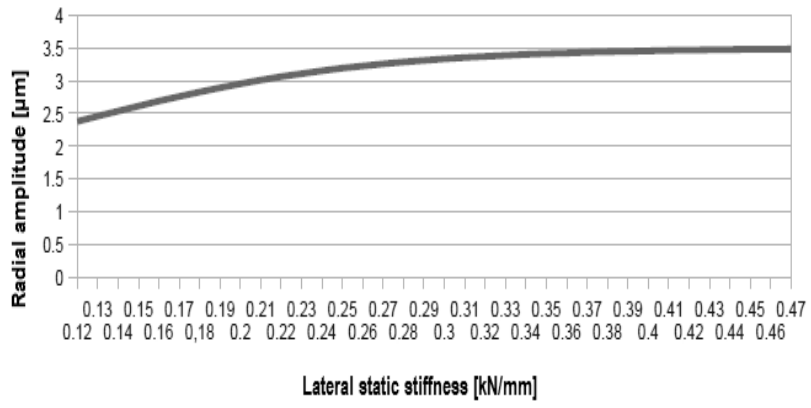

Fig.9c. Dependence of the radial amplitude on lateral static stiffness.

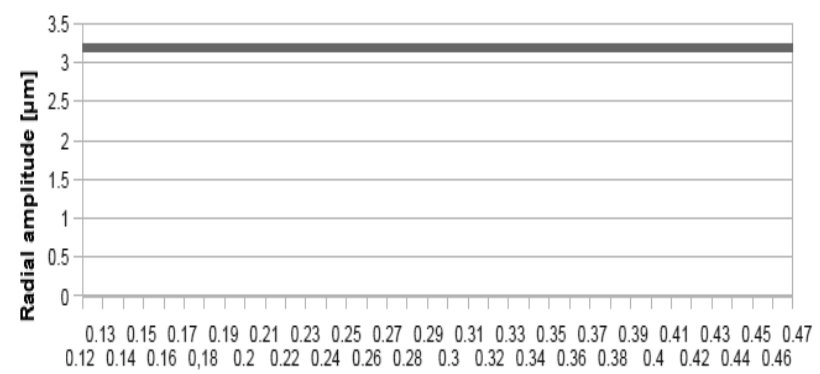

Static torsional stiffness [kNm/\%]

Fig.9d. Dependence of the radial amplitude on static torsional stiffness.

Results of the sensitivity analysis also clearly show the fact, that the used design influences neither radial frequency nor radial amplitude. Results for radial amplitude and frequency versus different tire profiles are shown in Fig.10. All these results were obtained by application of an independent software packet developed for industry.

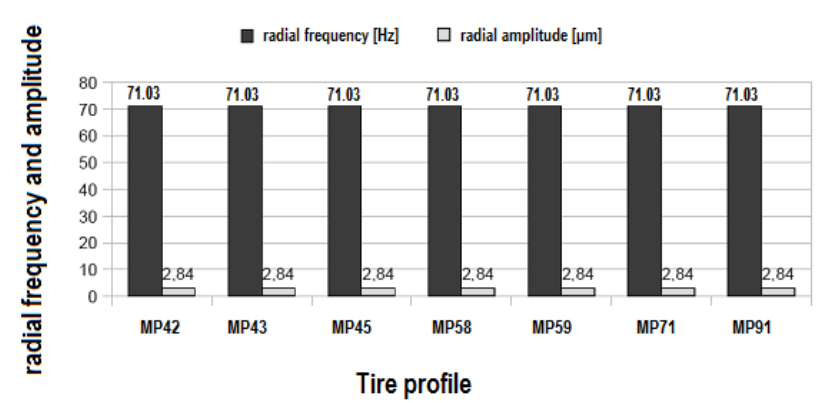

Fig.10. Radial amplitude and radial frequency versus different tire profiles.

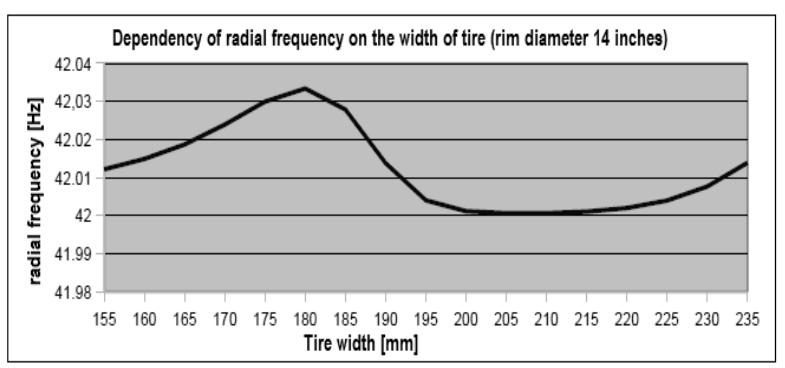

Fig.11a. Radial frequency versus rim diameter (14 inches). 


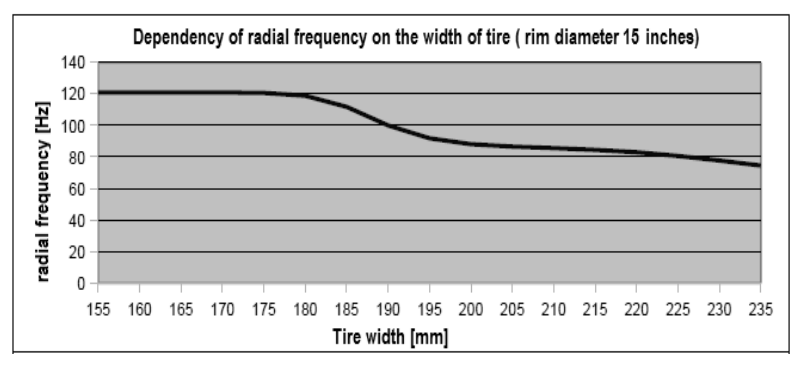

Fig.11b. Radial frequency versus rim diameter (15 inches).

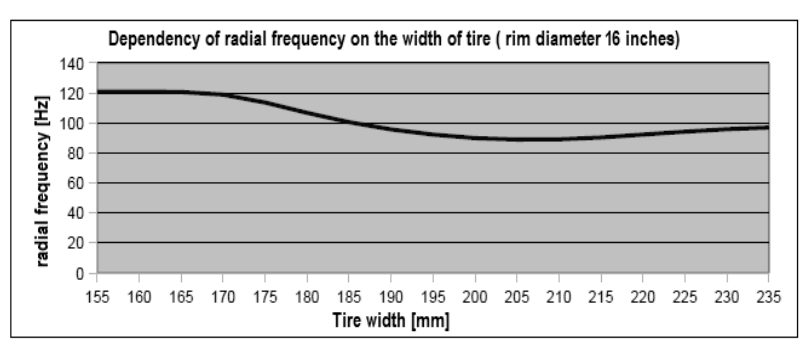

Fig.11c. Radial frequency versus rim diameter (16 inches).

Figs.11. present results of radial amplitude versus different rim diameter and tire width. Comparative statistical criteria for frequency prediction were as follows: SSE $=1265.871$, $R M S=4.314, R^{2}=0.976$.

It is clearly seen very weak dependence of the radial frequency versus tire with for tires of 14 inches (Fig. 11.a.). In last two cases (Figure 11b. and 11.c.) the same kind of dependences is more significant.

\section{CONCLUSION}

The variety of tire mode changes caused by changes in tire construction and inflation has been intensively studied by ESPI. Results are qualitatively comparable with those of FEM simulation.

The functional neuronal model was created for prediction of $O F$ tire oscillations. The model is capable of providing satisfactory prediction of frequency of tires with a mean square error $R M S=4.314 \mathrm{~Hz}$. The presented results and the experience acquired with applications of neural networks in material research show that their utilization in this area is very promising. Application of $A N N$ in modal analysis of tires is a very useful tool for tire designers.

It has been shown that the optical ESPI method has the advantages of non-contact and full field tire measurements. Because ESPI uses video recording and display, it works in real time to measure dynamic displacement, which enables implementation of this technique for tire vibration measurements.

\section{ACKNOWLEDGMENT}

This paper was created in the project No.Cz.1.05/2.1.00/01.0040 "Regional Materials Science and Technology Centre" within the frame of the operation program "Research and Development for Innovations" financed from the Structural Funds and from the state budget of the Czech Republic.

This research was also financed from projects labelled as InterDV,CZ.1.07/2.2.00/15.0132, and MPO TIP FRTI3/818, MPO, TIP FR-TI1/319. This paper has been elaborated in the framework of the IT4Innovations Centre of Excellence project, reg. no. CZ.1.05/1.1.00/02.0070 supported by Operational Program 'Research and Development for Innovations' funded by Structural Funds of the European Union and state budget of the Czech Republic.

\section{REFERENCES}

[1] Kováč, I., Krmela, J., Bakošová, J. (2011). Parametrizing of material input for modal analyses of FEA tire models. Metallurgical Journal, 64 (7), 73-78.

[2] Gaul, L., Willner, K., Hurlenbaus, S. (1999). Determination of material properties of plates from modal ESPI measurements. In 17th International Modal Analysis Conference (IMAC), 8-11 February 1999. Orlando, Florida: Society for Experimental Mechanics, 1756-1762.

[3] Caldersmith, G.W. (1984). Vibrations of orthotropic rectangular plates. Acta Acustica united with Acustica, 56 (2), 144-152.

[4] Kim, D.W., Lee, N.K., Na, N.K., Kwon, D. (2002). Mapping of surface residual stress field by laser interferometry using stress relaxation method. MRS Proceedings, 750, Y8.7.

[5] Jones, R., Wykes, C. (1986). Holographic and Speckle Interferometry, Second Edition. Cambridge University Press, 165-196.

[6] Špička, I., Heger, M., Franz, J. (2010). The mathematical-physical models and the neural network exploitation for time prediction of cooling down low range specimen. Archives of Metallurgy and Materials, 55 (4), 921-926.

[7] Frischer, R., David, J., Vrožina, M. (2011). Neural networks usage at crystallizers diagnostics. In Proceedings of METAL 2011, 18-20 May 2011. Brno, Czech Republic: Tanger Ltd, 134-138.

[8] Myllykoski, P., Larkiola, J., Nylander, J. (1996). Development of prediction model for mechanical properties of batch annealed thin steel strip by using artificial neural network modelling. Journal of Materials Processing Technology, 60 (1-4), 399-404.

[9] Jančíková, Z., Roubíček, V., Juchelková, D. (2008). Application of artificial intelligence methods for prediction of steel mechanical properties. Metalurgija, 47 (4), 339-342.

[10] Lee, Ch.Y., Hsieh, Y.H. (2012). Bearing damage detection of BLDC motors based on current envelope analysis. Measurement Science Review, 12 (6), 290295.

[11] Koštial, P., Jančíková, Z. (2012). Chosen methods of experimental data evaluation. In Proceedings of the 11th Youth Symposium on Experimental Solid Mechanics, 30 May - 2 June 2012. Brasov, Romania, 55-60. 